\title{
GANS: A Signalling Framework for Dynamic Interworking between Heterogeneous Networks
}

\author{
Nadeem Akhtar, Rui Campos, Cornelia Kappler, Pekka Pääkkönen, Petteri Pöyhönen, Di Zhou
}

\begin{abstract}
There is a growing trend towards convergence of telecommunication and data networks in order to support a richer set of services and applications. At the same time, increasing diversity and density of network access technologies has made the goal of providing connectivity anytime and anywhere a real possibility. Another important development is the emergence of small, low-complexity user owned networks, such as Personal Area Networks and Body Area Networks. Dynamic interworking, also known as network composition, between networks of different types and sizes is essential in the push towards convergence, as well as to realize truly seamless connectivity between heterogeneous access networks. Dynamic interworking requires signalling between different elements of the control planes of the different networks in order to coordinate the control functions and resources of the networks concerned. In this paper, we present the Generic Ambient Network Signalling protocol suite to address the diverse signalling requirements for dynamic interworking of networks.
\end{abstract}

Index Terms- Convergence, Dynamic Interworking, Control signalling, GANS, NSIS

\section{INTRODUCTION}

Convergence of telecommunication networks and the Internet has become very important, as the traditional divide between these networks is getting increasingly blurred. On the one hand, the customer base of VoIP services is expanding rapidly. On the other hand, mobile communication operators are pushing more and more web-based services into mobile handsets. The all-IP networking paradigm has been a key enabler of integration of telecommunication and data networks.

A parallel trend is the so-called plug and play networking or dynamic interworking of networks [1], [3], [11]. Nowadays, it is common for users to own a number of communication devices, such as laptops, mobile phones, and PDAs etc. In many cases, these devices are organised to form small low-

Nadeem Akhtar is with the University of Surrey, UK (email: N.Akhtar@surrey.ac.uk).

Rui Campos is with INESC Porto, Portugal (email: rcampos@inescporto.pt).

Cornelia Kappler, is with Siemens Communications, Berlin, Germany. (email: cornelia.kappler@siemens.com).

Pekka Pääkkönen, is with VTT Technical Research Centre of Finland, Oulu, Finland (email: Pekka.Paakkonen@,vtt.fi).

Petteri Pöyhönen is with Nokia, Helsinki, Finland (email: petteri.poyhonen@nokia.com).

Di Zhou is with PSE/Siemens, Vienna, Austria (email: di.zhou@siemens.com).

Note: Names of co-authors have been listed in alphabetical order. complexity networks, such as Body Area Networks, Personal Area Networks and Home Area Networks. In future, these networks are envisaged to interwork with each other and with access networks which, in turn, interwork with core networks. Dynamic interworking is being investigated in the Ambient Networks project [2] and the concept network composition has been proposed as a means of enabling convergence and dynamic interworking between networks of different types and sizes [3], [11]. Network composition is not limited to simply enabling routing across different network domains or roaming agreements between network operators; it aims to enable cooperative networking via sharing of control as well as resources, both physical and logical.

An important requirement for composition-based dynamic interworking is the coordination of control spaces. This may involve coordination of address spaces and addressing schemes, delegation/sharing of control over resources, merging or synchronization of network and policy databases etc. In order to establish the parameters that govern such a high level of coordination, a non-trivial amount of control signalling is needed between different entities in the networks involved. This paper proposes a generic signalling protocol suite for solving this problem; that is, we argue that signalling protocols for control space coordination - while encompassing a wide variety of different tasks - also always have some aspects in common. These commonalities merit a layering of the signalling protocols in a lower, common layer, and an upper task-specific layer.

There are already a number of protocols designed for control signalling between network elements. The Session Initiation Protocol (SIP) [4] is a flexible, general-purpose protocol that supports signalling for creating, modifying, and terminating sessions. Although SIP is independent of underlying transport protocols and the type of session being established, it is designed to run as an end-to-end protocol between user applications at end hosts. The Diameter protocol consists of a 'base' protocol and set of application protocols [5]. The Diameter base protocol has been designed to provide an Authentication, Authorization and Accounting (AAA) framework for applications such as network access or IP mobility. The protocol is generic and extensible in the sense that Diameter client protocols can be added on top of the base layer. However, these clients use the base protocol for signalling specifically related to AAA functions. The NSIS 
(Next Steps In Signalling) protocol suite [6] is being developed for signalling information pertaining to data flows. The aim is to support different signalling applications that need to install and/or manipulate flow-related control state in the network. The NSIS suite has a lower transport layer that provides common functions such as session management, peer discovery, secure channel establishment etc. On top of the transport layer reside a set of signalling applications for diverse control signalling, such as QoS control, middlebox communications etc.

Among the signalling protocols mentioned above, both SIP and NSIS are generic and extensible but they are mainly geared towards providing signalling support for data flows. Diameter is extensible but its scope is limited to AAA-related signalling. Control signalling required for dynamic interworking requires a much more flexible approach, including a wider set of signalling applications as well as a broader operational scope.

The generic and extensible protocol suite we propose in this paper is based on the NSIS protocol suite. However, there are significant differences between the two. Most importantly, unlike NSIS, the scope of our solution also includes signalling that may have no direct relation to any underlying data flows. Generic Ambient Network Signalling (GANS) is a suite of signalling protocols designed to enable efficient message exchange between related control functions of the control spaces of different networks. It supports the diverse signalling required for supporting dynamic interworking and convergence of heterogeneous networks.

The rest of this paper is organised as follows. In Section II, we introduce a set of use cases for GANS which illustrate the common functions that must be provided by the lower signalling layer. Section III presents an overview of GANS design and architecture. In Section IV, the different components of the protocol suite are described in detail. Finally, in Section V, we present conclusions and directions for future work.

\section{Gans Usage Scenarios}

When two networks interwork, they need to coordinate their control functions. The most basic of these control functions are addressing and routing. In today's IP networks, IP addresses usually are static, autoconfigured or assigned by a DHCP server. New nodes join a network on an individual basis and must adopt the addressing scheme being used in the network. However, when an entire network joins another network, e.g. two Personal Area Networks (PANs) merge, it is less clear whose addressing scheme should be used. Let us assume both networks use DHCP servers to allocate non-global addresses. In this case, the two networks need to agree on whether one of the DHCP servers should be disabled, or whether both should continue to run. In the latter situation, the address spaces from which the DHCP servers allocate addresses must be configured to be non-overlapping. The coordination of DHCP servers can be performed by the servers themselves, or by central entities representing the two networks. Either way, coordination of
DHCP servers is a typical problem that could be solved by the GANS signalling between the two networks. Besides the actual signalling semantics, the problem that needs to be solved is that e.g. DHCP Server A in network A initiating the coordination must locate its corresponding entity DHCP Server B in network $\mathrm{B}$, and establish a secure signalling relation with it (see Figure 1). Clearly, with the coordination of the DHCP servers the addressing problem is not yet solved. For example, duplicate addresses must be detected and new addresses must be assigned. However, this problem must be solved within DHCP scope rather than with the GANS protocol.

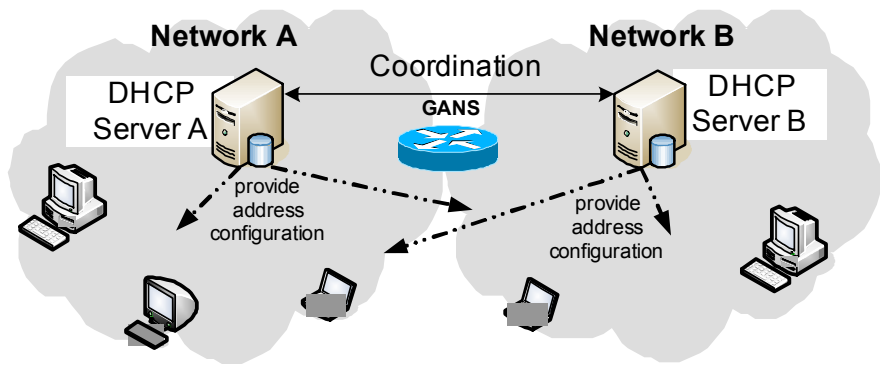

Figure 1: DHCP Server coordination

Another well-known scenario is that of control coordination between networks is the negotiation of Service Level Agreements (SLAs). Here, two network entities, e.g. bandwidth brokers, negotiate the QoS - bandwidth, priority etc. - that will be provided to user traffic originating from the other network. As above, the bandwidth brokers need to find each other and establish a secure signalling relationship. When SLAs are negotiated in today's networks, the relation between the bandwidth brokers usually is statically configured. However, in future dynamic network scenarios, with entire networks on the move, this will no longer be feasible. GANS therefore includes functionality for dynamically locating signalling peers and for securing their signalling relationship. Note that we can illustrate the problem with the same Fig. 1 above, by replacing "DHCP Server" by "Bandwidth Broker".

The last scenario concerns the case of two networks trying to discover information on each other. One could picture a database in each network that provides information on linklayer technologies supported, protocols used etc, similar to the Media-Independent Information Service defined in IEEE 802.21 [9]. Before the networks start interworking they could consult each other's database to find out whether they are compatible. Also this communication could be performed by means of the GANS protocol.

In addition to the specific requirements of each signalling application above, the problems common to all three examples:

- Localization of the signalling peer

- Establishing a secure signalling relation

- Transporting signalling messages between signalling peers. 


\section{Gans Overview}

\section{A. Design Approach}

The usage scenarios presented above show both common and specific requirements. On the one hand, every scenario illustrates a signalling application in a specific field requiring specific handling and processing. On the other hand, all the scenarios require basic and efficient data transport connections enabling transmission of various signalling messages among diverse networks, and meeting general and specific security and quality requirements. This leads to a design approach based on a two layer model: a signalling layer consisting of individual protocol entities handling special needs of each specific application field, and a transport layer providing a general message delivery service ensuring efficient and secure signalling data transportation among cooperating networks. This approach is consistent with the architectural approach of the IETF working group NSIS protocol suite [6]. In fact, GANS generalizes and extends the NSIS protocol suite.

NSIS is a protocol suite for manipulating control state in network elements on the path of a data flow. Examples of NSIS signalling are protocols for reserving QoS [8], configuring Network Address Translation entities and firewalls [7]. A future application may be a protocol for configuring metering entities [12]. Such diverse applications are supported by defining two layers. The lower layer, NTLP (NSIS Transport Layer Protocol), takes care of tasks common to all signalling applications: finding the neighbour signalling peer, establishing a security relation with this peer, and transporting messages to upstream and downstream adjacent peers. While in principle several NTLPs are conceivable, the only NTLP currently defined is called GIST [10]. The upper NSIS layer consists of the different signalling applications such as those enumerated above. The signalling application protocols, NSLPs (NSIS Signalling Layer Protocols) define the signalling semantics that are specific to the requirements of each NSLP. They form a message and pass it to the NTLP for delivery.

GANS adopts the NSIS two-layer approach as shown in Figure 2. This paper focuses on the lower GANS layer, GTLP (GANS Transport Layer Protocol). Our design is a backwardcompatible extension of the NSIS lower layer, GIST.

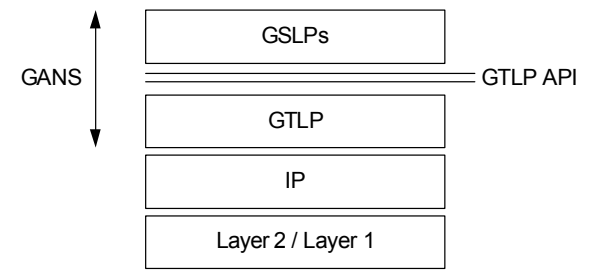

Figure 2: Two layer GANS model

The service GTLP offers to the signalling applications (referred to as GANS Signalling Layer Protocols or GSLPs) includes finding the neighbour signalling peer, establishing a security relationship with it, and transporting messages, just as NTLP. The difference with NTLP is in the location of the signalling peer. In NSIS, the peer is found by exploiting the fact that it must be the next suitable NSIS hop on the data path towards the destination of the flow. In GANS, the signalling is not always flow related, and the IP address of the signalling peer is not always known either. Rather, it is known that the signalling peer is located in a specific network, and that it fulfils a certain role, e.g. "DHCP server" or "bandwidth broker". The GANS signalling application on top of GTLP therefore just addresses the message to a symbolic name, e.g. "DHCPServer.networkX". The main challenge for GTLP is then to resolve the role-based identifier into a communication address, e.g., an IP address; this resolution is transparent to application. Our protocol design focuses on the signalling aspect and assumes that an infrastructure exists for resolving the symbolic name. We make very few assumptions about this infrastructure, e.g. DNS might be an option. Furthermore, the namespace used to create symbolic names is beyond the scope of GANS design. The GANS name resolution clearly is an alternative to anycast addressing.

\section{B. GANS Architectural Components}

The architecture of GANS protocol suite is shown in Figure 3. GANS reuses as much as possible the protocol components and entities of NSIS and ensures backward compatibility to NSIS in the sense that NTLPs are able to run without any modification in the GTLP environment, and networks keep working without any additional modification when some NTLP implementations are replaced by GTLP implementations.

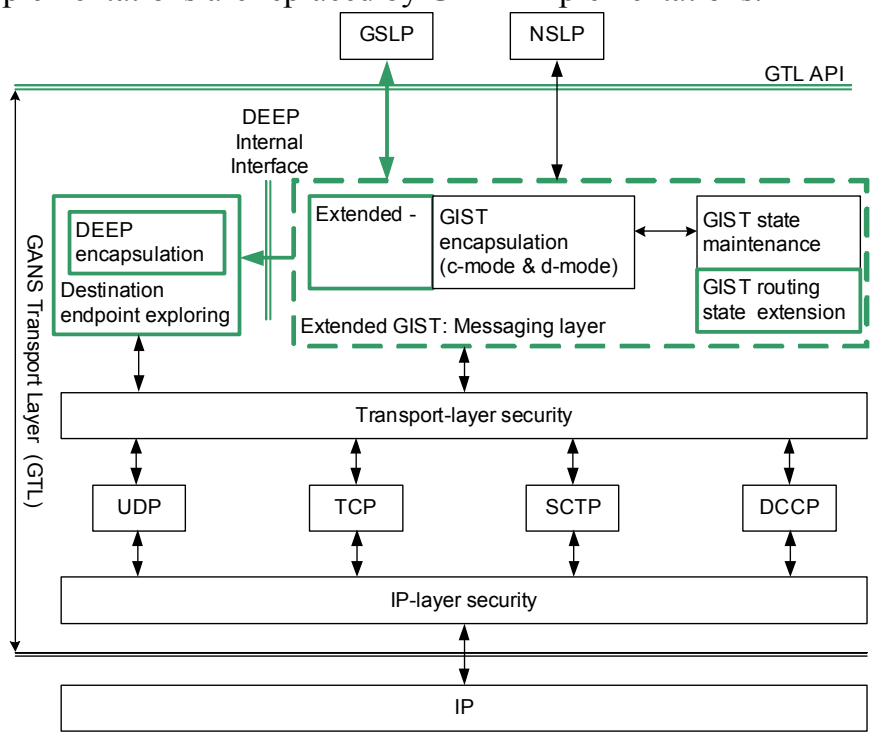

Figure 3: GANS Architecture

\section{Protocol Design}

GTLP comprises two main building blocks: the Extended General Internet Signalling Transport (EGIST), which is based on the GIST and the Destination Endpoint Exploration Protocol (DEEP). EGIST provides the actual transport framework for the GSLP messages received through the GTLP API. DEEP [13] is used for name resolution when the signalling 
application addresses the destination endpoints with symbolic names.

When GTLP receives a message from a GSLP from the API, if symbolic name is used, it is processed and passed to DEEP. Using the name resolution infrastructure, DEEP returns the communication address of the destination endpoint to which the message should be sent. Thereafter, a message association is set up between the two signalling endpoints and then the EGIST module encapsulates the GSLP message and sends it to the contact address returned by DEEP. Depending on the information received from GSLP (along with the message) and local policies, EGIST will either use an unreliable or reliable transport protocol to send the message. If desired, the two nodes also establish a security association based on the mechanism specified in [10]. The message association is reused for any messages that follow after the first one.

In the following, DEEP and EGIST are presented in more detail, and a brief description of the GTLP API is provided. We also briefly mention the GANS signalling layer.

\section{A. GTLP API}

GTLP API is the interface provided by GTLP to GSLPs. It is a simple extension of and backward compatible to GIST API [10], so that applications running on top of GIST can also run over GTLP without any modification. Basically, the parameters and primitives defined for the GIST API are re-used. The same interface is presented at every GTLP node. However, applications may invoke it differently at different nodes depending on local policy and needs of signalling applications.

\section{B. Extended GIST}

The EGIST protocol is the actual transport framework provided by GTLP to the signalling applications. As mentioned before, EGIST is derived from the GIST protocol. It comprises all functionality already deployed by GIST plus additional functionality required for the GANS protocol suite. EGIST uses the underlying protocols such as TCP, UDP and Transport Layer Security protocol to provide the transport and security services that are requested by the GSLP messages. Interactions between EGIST and GSLPs occur via the GTLP API, while interactions between EGIST and DEEP occur via an internal interface. The new functionalities that EGIST adds on top of those provided by GIST are:

- Support for addressing signalling endpoints by names

- Interface with DEEP

- Name binding state storage and maintenance

Concerning the way signalling messages are routed towards the proper adjacent peer, GIST defines the concept of Message Routing Method (MRM) [10]. The MRM loosely defines the algorithm for discovering the route that the signalling messages should follow between the source and destination at the GIST layer. GIST design supports multiple MRMs; default is the "path-coupled" MRM which supports routing of messages along the path of data flow. Signalling applications indicate to EGIST the MRM to be used for message forwarding.

To support use of symbolic names and signalling that is not tied to any underlying data flow, a new MRM is defined by EGIST. It requires modifications with respect to the internal processing of the messages received from the signalling applications, namely regarding the way messages are routed and the way the two operation modes (connection mode and datagram mode) defined by GIST are used.

Support for symbolic names to address destinations requires the storage of the mapping between names and the corresponding IP addresses provided by DEEP. DEEP is a stateless protocol, i.e., it does not maintain any state information. Therefore, EGIST is the entity responsible for maintaining such state information.

A further extension to GIST is related to the update of a name binding state. When there is a relocation of signalling endpoint, which causes a change in the IP address and/or in the port being used by one of the signalling endpoints participating in a signalling session, there is a need for updating the corresponding name binding state. EGIST is responsible for such update, as it is the entity storing the name binding state. Further details about EGIST can be found in [14].

\section{DEEP}

DEEP supports the distributed resolution of a symbolic name into a communication address. We propose a sequential method in which the symbolic name is resolved in several steps, by forwarding the same DEEP message with updated name resolution status information from one resolver to the next until the IP address of the relevant node is discovered.

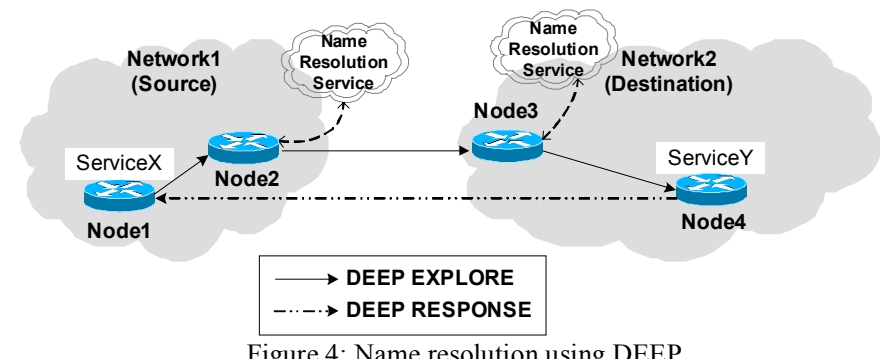

For example, consider the scenario in Figure 4 where Node1 in Network1 wants to resolve the name of the node offering Service1 in Network2. Node 1 sends a DEEP EXPLORE message with the symbolic name ServiceY.Network2 to a name resolving node (Node2), local to Network1. This node may either know the IP address of the name resolver (Node3) in Network2, or the IP address of an intermediary name resolver that is able to resolve a part of the current symbolic name towards the complete name resolution through the use of the sequential procedure aforementioned. Upon knowing the IP address of the next resolver, the current DEEP node forwards the EXPLORE message there; this process is sequentially repeated until the name resolution process reaches the name resolver in Network2. The name resolver in Network2 knows the IP address of the contact point for ServiceY (Node4). At this point, the name is fully resolved and the so-called DEEP RESPONSE message is sent back to the originating node (Node1). With this method, it is possible for networks to let a central 'Signalling Gateway' handle all incoming GANS messages, e.g., in order to hide its internal infrastructure. This can be achieved by the local name resolver always returning the IP address of the Signalling Gateway. It is also possible, 
however, to return the IP address of more specific nodes, thereby distributing the load more evenly.

Rather than using the DEEP protocol for name resolution, in principle, the DNS infrastructure could be used. However, DNS is not a sufficient standalone option within the GANS scope, as it is not able to react quickly enough to dynamic changes of name address mappings like Communication Endpoint-to-physical-node mappings. Furthermore, DNS is not well-suited in situations where connectivity to core network is not available and the use of various infrastructure services is not possible.

It is transparent to DEEP how local name resolution services are handling name resolution information like caching it locally for further use. DEEP provides internal name resolution services for GTLP via a unified internal interface, which hides the implementation of the local name resolution service. This internal interface is actually used by EGIST upon receiving a message from a signalling application and when no name binding state information exists locally with respect to the destination symbolic name specified by the application. Externally, DEEP uses mainly two interfaces: a local name resolution interface and a message transport service interface. At this moment, DEEP is specified to run over UDP, but it can be used with other transport protocols as well.

Although the Service Location Protocol [16] has also been proposed for the discovery of network services, clearly it is more suited to scenarios where users/hosts want to discover a service with certain attributes within a network, typically local area networks. DEEP, on the other hand, provides the functionality to locate services across network/domain/namespace boundaries utilising the available name resolution mechanisms such as DNS, LDAP etc.

\section{GANS Signalling Layer}

The upper layer of the GANS protocol suite consists of a set of GSLPs which define the protocols for signalling related to specific controls functions. For example, a GSLP has been designed to establish Service Level Agreements between networks and additional GSLPs are foreseen. In general, the control messages of signalling applications may or may not be coupled to an underlying data flow; both types of applications are supported by GANS. From the signalling applications point of view, the two-layer structure has the advantage of making them independent of the protocol stack below GTLP.

\section{Conclusions}

We presented GANS which is a generic and extensible protocol suite to support control signalling in dynamic and heterogeneous networking environments. Use cases were presented that underlined the problems common to our signalling scenarios, motivating the introduction of a twolayered approach based on NSIS. The protocol architecture was presented and the role of different components explained, especially the GANS transport layer. GTLP is not an end in itself. Rather, it is meant to be used by signalling applications. Performance evaluation of both DEEP and EGIST has been carried out using simulations [14][15].

\section{ACKNOWLEDGMENT}

The authors would like to thank Robert Hancock for enlightening discussions on GIST.

This work is supported by the Ambient Networks Project, partially funded by the European Commission under its Sixth Framework Programme. It is provided "as is" and without any expressed or implied warranties, including, without limitation, the implied warranties of fitness for a particular purpose. The views and conclusions contained herein are those of the authors and should not be interpreted as necessarily representing the official policies or endorsements, either expressed or implied, of the Ambient Networks Project or the European Commission.

\section{REFERENCES}

[1] R. Campos et al., "Dynamic and Automatic Interworking between Personal Area Networks using Composition", in Proc. 16th IEEE International Symposium on Personal Indoor and Mobile Radio Communications, September 2005, Berlin, Germany.

[2] IST Ambient Networks Project, http://www.ambient-networks.org

[3] N. Niebert et al., Ambient Networks: An Architecture for Communication Networks beyond 3G, IEEE Wireless Communications, vol. 11, pp.14-22, April 2004.

[4] J. Rosenberg, H. Schulzrinne et al, "SIP: Session Initiation Protocol", RFC 3261, June 2002.

[5] P. Calhoun, J. Loughney, Erik Guttman, G. Zorn and J. Arkko, "Diameter Base Protocol", RFC 3588, September 2003.

[6] R. Hancock, G. Karagiannis, J. Loughney and S. van den Bosch, "Next Steps in Signalling (NSIS): Framework", RFC 4080, June 2005.

[7] M. Stiemerling, H. Tschofenig and C. Aoun, "NAT/Firewall NSIS Signalling Layer Protocol (NSLP)", draft-ietf-nsis-nslp-natfw-11, Internet Draft (work in progress), April 2006.

[8] J. Manner, G. Karagiannis, A. McDonald and S. Van den Bosch, "NSLP for Quality-of-Service Signalling”, draft-ietf-nsis-qos-nslp-10, Internet Draft (work in progress), March 2006.

[9] "Draft IEEE Standard for Local and Metropolitan Area Networks: Media Independent Handover Services", IEEE LAN/MAN Draft IEEE P802.21/D00.01, July 2005.

[10] H. Schulzrinne and R. Hancock, "GIST: General Internet Signalling Transport”, draft-ietf-nsis-ntlp-09, Internet Draft (work in progress), Februrary 2006.

[11] C. Kappler, P. Mendes, P. Pöyhönen, C. Prehofer and D. Zhou, "A Framework for self-organized Network Composition", Proc. 1st IFIP TC6 WG6.6 International Workshop on Autonomic Communication (WAC 2004), Springer LLNC-IFIP Series Vol. 3547., Berlin, Oct. 2004.

[12] Fessi, C. Kappler, C. Fan and A. Klenk, Framework for Metering NSLP, draft-fessi-nsis-m-nslp-framework, Internet Draft (work in progress), November 2005.

[13] P. Pöyhönen et al., "DEEP - A Generic Name Resolution Protocol for Heterogeneous Networks", Proc. IEEE International Conference on Information \& Communication Technologies: from Theory to Applications - ICTTA'06, April 2006.

[14] R. Campos et al., "On the Evaluation of the Extended Generic Internet Signalling Transport Protocol", Proc. 15 ${ }^{\text {th }}$ IST Mobile Summit, June 2006.

[15] P. Pääkkönen et al., "Scalability of Name Resolution for Ambient Networks", Proc. 4th International Conference on Wired/Wireless Internet, Bern, May 2006.

[16] E. Guttman, C. Perkins, J. Veizades, M. Day, "Service Location Protocol, Version 2", Internet RFC2608, June 1999. 